\title{
Benefícios da Talassoterapia e Balneoterapia na Fibromialgia
}

\section{Benefits of Thalassotherapy and Balneotherapy for Fibromyalgia}

\author{
Sandra Cristina de Andrade( ${ }^{(1)}$, Ranulfo Fiel Pereira Pessoa de Carvalho ${ }^{(2)}$, \\ Aluízio Silvio Soares ${ }^{(3)}$, Maria José Vilar ${ }^{(4)}$
}

\section{RESUMO}

Talassoterapia e balneoterapia são modalidades terapêuticas utilizadas há vários anos em outras regiões do mundo na prevenção e no tratamento de diversas enfermidades, incluindo doenças reumáticas. No entanto, só recentemente têm sido descritas na literatura em fibromialgia (FM), contribuindo para a redução da dor e de outros sintomas da doença e melhorando a qualidade de vida dos pacientes. Nesta revisão são relatados os principais estudos que avaliam a talassoterapia e/ou a balneoterapia como abordagem terapêutica na FM, abordando aspectos a serem investigados no intuito de estabelecer o valor dessa forma de tratamento. Os autores ainda destacam a necessidade da realização de estudos no Brasil, utilizando principalmente a talassoterapia, uma vez que o baixo custo, aliado ao fácil acesso de boa parte das pessoas ao litoral, podem beneficiar pacientes com FM.

Palavras-chaves: fibromialgia, talassoterapia, balneoterapia.

\section{INTRODUÇÃO}

A fibromialgia (FM) é uma síndrome reumática crônica não-inflamatória, de etiopatogenia desconhecida, que acomete preferentemente mulheres, caracterizada pela presença de dor musculoesquelética difusa e dos chamados tender points ${ }^{(1)}$. A dor, juntamente com outras características, como distúrbio do sono e fadiga, contribuem para a piora da aptidão cardiorrespiratória, do estado geral de saúde e da qualidade de vida dos pacientes $^{(2-5)}$. A abordagem terapêutica freqüentemente utilizada na FM é focalizada no alívio dos sintomas: analgésicos, antidepressivos, exercícios e educação do paciente são amplamente empregados. Entretanto, essas medidas, quando aplicadas isoladamente, não conseguem melhorar significantemente

\section{ABSTRACT}

Thalassotherapy and balneotherapy are therapeutic strategies commonly used in other countries for the prevention and treatment of several diseases, including rheumatic diseases. However, only in the recent years its use in the treatment of fibromyalgia (FM) has been reported. The potential beneficial effects include the reduction of pain and other related symptoms of FM, thus improving the quality of life. The authors revise studies focusing on thalassotherapy and/or balneotherapy in the treatment of FM, discussing their benefits and methodological biases that still preclude a more precise evaluation on the efficacy of these methods. Finally, the authors emphasize the need for such studies in Brazil, mainly thalassotherapy, since the low cost and good accessibility to sea, might well provide benefit to our patients.

Keywords: thalassotherapy, balneotherapy, fibromyalgia.

a qualidade de vida dos pacientes. Isso realça a necessidade de terapias mais efetivas, no intuito de mais bem atender às demandas de saúde do paciente com $\mathrm{FM}^{(6,7)}$.

Apesar de os exercícios aeróbicos serem citados como a intervenção de reabilitação física que promove maior ganho na diminuição do impacto dos sintomas da $\mathrm{FM}^{(8,9)}$, diversas modalidades terapêuticas surgiram como alternativas (terapias manuais, osteopatia, quiropraxia, acupuntura, massagens, ioga, watsu, tai chi, talassoterapia e balneoterapia) que podem contribuir no tratamento da doença ${ }^{(10-16)}$. Terapias em estâncias termais utilizando a balneoterapia e a talassoterapia são freqüentemente utilizadas em vários países da Europa e do Oriente Médio para tratamento da FM, bem como de outras doenças reumáticas, desde muitos anos ${ }^{(16-21)}$.

Recebido em 16/1/2008. Aprovado, após revisão, em 19/3/2008. Declaramos a inexistência de conflitos de interesse.

1. Fisioterapeuta, doutoranda do Programa de Pós-Graduação em Ciências da Saúde da Universidade Federal do Rio Grande do Norte (UFRN); Professora do Curso de Fisioterapia da Universidade Potiguar (UnP).

2. Fisioterapeuta aluno de Especialização na Universidade Gama Filho (UGF).

3. Fisioterapeuta aluno de Especialização na Faculdade Integrada do Recife (FIR).

4. Professora Doutora da Disciplina de Reumatologia e do Programa de Pós-Graduação em Ciências da Saúde da UFRN.

Endereço para correspondência: Sandra Cristina de Andrade, Rua São Cristóvão, 3831, apto. 101, Lagoa Nova, 59056-290, Natal, RN, e-mail: sandaandrade@unp.br/ sandra.andrade.fisio@gmail.com 
A talassoterapia consiste de modalidade terapêutica que combina banho na água do mar, clima marinho e radiação solar. Algumas regiões do mundo não apresentam condições geográficas ou climáticas compatíveis com a realização da talassoterapia, existindo assim um crescente interesse, principalmente nos países da Europa, na criação de centros de balneoterapia nas proximidades das costas marítimas para oferecer essa modalidade de tratamento. A balneoterapia é definida como tratamento por meio de banhos de qualquer origem, podendo ser com a água do mar, não necessariamente inserido no ambiente marítimo, havendo controle do ambiente de tratamento e preservação dos potenciais princípios terapêuticos da água do $\operatorname{mar}^{(22,23)}$. No Brasil, a balneoterapia é praticada em alguns locais no litoral de Santa Catarina, Ceará e Bahia, habitualmente em ambientes de alto custo, com características de centros prioritariamente recreativos.

\section{PRocedimentos DE Coleta}

Foi realizada revisão da literatura utilizando as bases de dados Lilacs e Medline de 1990 a 2008. As palavras-chaves utilizadas foram: fibromyalgia, thalassotherapy e balneotherapy e as similares em português. Foram excluídos artigos não escritos em inglês ou português.

\section{TALASSOTERAPIA E BALNEOTERAPIA NA FIBROMIALGIA}

Em reumatologia, há relatos de estudos que demonstram os benefícios da talassoterapia e da balneoterapia no tratamento de artrite reumatóide ${ }^{(24,25)}$, artrite psoriásica ${ }^{(26,27)}$, espondilite anquilosante ${ }^{(28,29)}$, osteoartrite ${ }^{(30,31)}$, algias de coluna $^{(32,33)}$ e FM $^{(16,34-40)}$. No entanto, evidências científicas não são suficientes para estabelecer, de maneira definitiva, a aparente eficácia da talassoterapia e da balneoterapia em doenças reumáticas. Revisões sistemáticas Cochrane para avaliar a eficácia da balneoterapia na artrite reumatóide e na osteoartrite concluíram que, apesar de os achados positivos relatados na maioria dos estudos, esses devem ser vistos com cautela, em virtude da baixa qualidade metodológica e de análise estatística inadequada.

A Tabela 1 sintetiza os protocolos e os resultados dos principais estudos realizados nos últimos anos em pacientes com FM utilizando a talassoterapia e/ou a balneoterapia. A maioria dos estudos mostra efeitos benéficos da talassoterapia e da balneoterapia na FM, promovendo melhora da dor, da ansiedade, do distúrbio do sono, do número de tender points, dos índices de depressão e da qualidade de vida.
Em estudo aberto, incluindo 28 pacientes com artrite psoriásica associada à $\mathrm{FM}$, foram utilizadas duas modalidades de balneoterapia em dias alternados (banhos de lama e enxofre em piscina aquecida) e sessões diárias de talassoterapia (banhos de imersão no mar), durante quatro semanas. Ao final, foi observada diminuição significativa no número de tender points e melhora do limiar de dor (avaliada por dolorímetro), bem como diminuição da rigidez matinal e número de articulações dolorosas ${ }^{(34)}$.

Em estudo realizado por Zijlstra et al.(36), balneoterapia, exercícios e educação do paciente foram realizados em um grupo de 58 pacientes com FM e comparados com 76 pacientes de um grupo dito controle, que usaram tratamento medicamentoso domiciliar, seguindo a rotina diária. Os pacientes do grupo experimental realizaram durante duas semanas, hospedados em estância termal, os seguintes procedimentos: banho turco, pacotes quentes com algas, massagens sob chuveiro e hidromassagem (3 horas em dias alternados); nos outros dias realizaram uma hora de exercício em grupo (aquecimento, alongamentos e exercícios aeróbicos de baixa intensidade) e sessões de orientações educacionais. Os resultados mostraram melhora estatisticamente significante do grupo experimental na avaliação do estado de saúde pelo RAND 36-item health suvery (RAND-36) e na maioria dos outros parâmetros avaliados. Após três meses, a melhora clínica persistiu para o componente físico do RAND-36, mas não para o componente mental, não sendo observada diferença entre os grupos após 12 meses do início do estudo. Um padrão de melhora também foi observado após três e seis meses em alguns escores do Fibromyalgia Impact Questionnaire (FIQ), McGill Pain Questionnaire, Beck Depression Inventory (BDI) e no número de tender points. No entanto, não houve diferença entre os grupos no teste de 6 minutos de caminhada. Os autores concluíram que a combinação de balneoterapia, exercícios físicos e educação pode produzir melhora clínica em pacientes com FM e sugerem a necessidade de estudos com maior tempo de tratamento para avaliar o papel do condicionamento físico.

Recentemente, em estudo multicêntrico realizado em quatro estâncias termais na Itália, pacientes de cidades vizinhas que estavam sem resposta ao tratamento medicamentoso há, pelo menos, três meses, foram divididos aleatoriamente em dois grupos de 40 , a saber: aqueles do grupo experimental se dirigiam diariamente às estâncias para realizar sessões de balneoterapia (lama quente por 15 minutos e imersão em água termal por 10 minutos durante duas semanas) voltando à sua rotina após as sessões; os do 
TABELA 1

RESUMO DOS PROTOCOLOS E DOS RESULTADOS DOS PRINCIPAIS ESTUDOS REALIZADOS NOS ÚLTIMOS ANOS EM PACIENTES COM FIBROMIALGIA UTILIZANDO A TALASSOTERAPIA E/OU A BALNEOTERAPIA

\begin{tabular}{|c|c|c|c|c|c|c|}
\hline \multirow{2}{*}{$\begin{array}{l}\text { Autor } \\
\text { Ano }\end{array}$} & \multirow{2}{*}{$\begin{array}{l}\text { Número (n) } \\
\text { Experim. (E) } \\
\text { Controle (C) }\end{array}$} & \multirow{2}{*}{$\begin{array}{c}\text { Condutas } \\
\text { Experimental (E) }\end{array}$} & \multirow{2}{*}{$\begin{array}{c}\text { Condutas } \\
\text { Controle (C) }\end{array}$} & \multirow[t]{2}{*}{ Parâmetros avaliados } & \multicolumn{2}{|c|}{ Resultados } \\
\hline & & & & & Controle (C) & Experimental (E) \\
\hline $\begin{array}{l}\text { Buskila } \\
\text { et al. } \\
2001\end{array}$ & $\begin{array}{l}n=48 \\
(E=24) \\
(C=24)\end{array}$ & $\begin{array}{l}\text { Banhos de en- } \\
\text { xofre em piscina } \\
\text { aquecida. }\end{array}$ & Sem tratamento. & $\begin{array}{l}\text { EVA (dor, fadiga, ansie- } \\
\text { dade, depressão, rigidez, } \\
\text { bem-estar geral) + presen- } \\
\text { ça ou ausência (distúrbio } \\
\text { no sono, cefaléia, pares- } \\
\text { tesia, SCI, "inchaço") + } \\
\text { FIQ + FDI + contagem TP + } \\
\text { limiar de dor dos TP }\end{array}$ & $\begin{array}{l}\text { Melhora significante (dor, } \\
\text { fadiga, rigidez, ansiedade) } \\
\text { redução na freqüência } \\
\text { (cefaléia, distúrbio no } \\
\text { sono, "inchaço") }\end{array}$ & $\begin{array}{l}\text { Melhora significante } \\
\text { de todos os parâ- } \\
\text { metros avaliados, } \\
\text { exceto limiar de } \\
\text { dor dos TP }\end{array}$ \\
\hline $\begin{array}{l}\text { Sukenik } \\
\text { et al. }{ }^{(34)} \\
2001\end{array}$ & $\begin{array}{c}n=28 \\
(E=28)\end{array}$ & $\begin{array}{l}\text { Banhos de en- } \\
\text { xofre }+ \text { argila }+ \\
\text { mergulhos no } \\
\text { mar + exposição } \\
\text { ao sol. }\end{array}$ & $\longrightarrow$ & $\begin{array}{l}\text { Rigidez matinal + número } \\
\text { de art. ativas + contagem } \\
\text { TP + limiar de dor dos TP }\end{array}$ & $\longrightarrow$ & $\begin{array}{c}\text { Melhora significante } \\
\text { em todos os parâ- } \\
\text { metros avaliados }\end{array}$ \\
\hline $\begin{array}{l}\text { Neumann } \\
\text { et al. }{ }^{(38)} \\
2001\end{array}$ & $\begin{array}{l}n=48 \\
(E=24) \\
(C=24)\end{array}$ & $\begin{array}{l}\text { Banhos em pisci- } \\
\text { na de enxofre. }\end{array}$ & Sem tratamento & $\begin{array}{l}\text { SF-36 + HAQ + EVA (dor, } \\
\text { fadiga, distúrbio no sono, } \\
\text { SCI, bem-estar geral) }\end{array}$ & $\begin{array}{l}\text { Melhora (dor, fadiga e } \\
\text { bem-estar geral) }\end{array}$ & $\begin{array}{c}\text { Melhora significante } \\
\text { da dor, fadiga, bem- } \\
\text { estar geral e SF-36 }\end{array}$ \\
\hline $\begin{array}{l}\text { Evcik } \\
\text { et al. } \\
2002\end{array}$ & $\begin{array}{l}n=42 \\
(E=22) \\
(C=20)\end{array}$ & $\begin{array}{l}\text { Banhos com a } \\
\text { água do mar em } \\
\text { piscina aquecida. }\end{array}$ & Sem tratamento & $\begin{array}{c}\mathrm{FIQ}+\mathrm{BDI}+\mathrm{EVA}(\text { dor })+ \\
\text { contagem TP }\end{array}$ & Sem alteração significante & $\begin{array}{c}\text { Melhora significante } \\
\text { em todos os parâ- } \\
\text { metros avaliados }\end{array}$ \\
\hline $\begin{array}{l}\text { Zijlstra } \\
\text { et al. }{ }^{(36)} \\
2005\end{array}$ & $\begin{array}{l}n=134 \\
(E=58) \\
(C=76)\end{array}$ & $\begin{array}{l}\text { Banhos com algas } \\
+ \text { massagens }+ \\
\text { exercícios no solo } \\
+ \text { orientações. }\end{array}$ & Sem tratamento & $\begin{array}{l}\text { RAND } 36 \text { + FIQ + McGill } \\
\text { + EVA (bem-estar geral, } \\
\text { distúrbio no sono) + BDI + } \\
\text { CIS + contagem TP + teste } \\
\text { de caminhada ( } 6 \text { minutos) }\end{array}$ & Sem alteração significante & $\begin{array}{c}\text { Melhora significante } \\
\text { em todos os } \\
\text { parâmetros } \\
\text { avaliados }\end{array}$ \\
\hline $\begin{array}{l}\text { Donmez } \\
\text { et al. }{ }^{(39)} \\
2005\end{array}$ & $\begin{array}{l}n=30 \\
(E=16) \\
(C=14)\end{array}$ & $\begin{array}{l}\text { Banhos (piscinas, } \\
\text { chuveiro térmico } \\
\text { com sódio, cloro, } \\
\text { bicarbonato, } \\
\text { fluoreto. }\end{array}$ & Sem tratamento & $\begin{array}{c}\text { EVA (dor, fadiga, sono, } \\
\text { bem-estar geral e SCI) + } \\
\text { contagem TP + limiar de } \\
\text { dor dos TP + FIQ + BDI }\end{array}$ & Sem alteração significante & $\begin{array}{c}\text { Melhora significante } \\
\text { no FIQ + dor + con- } \\
\text { tagem TP fadiga e } \\
\text { bem-estar geral }\end{array}$ \\
\hline $\begin{array}{l}\text { Ardiç } \\
\text { et al. }{ }^{(40)} \\
2006\end{array}$ & $\begin{array}{c}n=34 \\
(E=12) \\
(C 1=12) \\
(C 2=10)\end{array}$ & $\begin{array}{l}\text { Banhos com } \\
\text { águas de estân- } \\
\text { cias termais. }\end{array}$ & $\begin{array}{c}\text { C1 = Sem tratamento. } \\
\text { C2 = mulheres saudáveis }\end{array}$ & $\begin{array}{l}\text { EVA (dor) + contagem TP + } \\
\text { limiar de dor dos TP + BDI } \\
+ \text { FIQ + PGE2 + LTB4 + IL1 }\end{array}$ & Sem alteração significante & $\begin{array}{c}\text { Melhora significante } \\
\text { em todos os } \\
\text { parâmetros } \\
\text { avaliados }\end{array}$ \\
\hline $\begin{array}{l}\text { Fioravanti } \\
\text { et al. }{ }^{(37)} \\
2007\end{array}$ & $\begin{array}{l}n=80 \\
(E=40) \\
(C=40)\end{array}$ & $\begin{array}{c}\text { Banhos de lama + } \\
\text { banhos em água } \\
\text { quente. }\end{array}$ & Sem tratamento & $\begin{array}{c}\mathrm{FIQ}+\mathrm{HAQ}+\mathrm{AIMS} 1+ \\
\text { contagem TP + EVA (dor de } \\
\text { cabeça, fadiga, sono e SCI) }\end{array}$ & Sem alteração significante & $\begin{array}{c}\text { Melhora significante } \\
\text { em todos os parâ- } \\
\text { metros avaliados }\end{array}$ \\
\hline
\end{tabular}

SCI = síndrome do cólon irritável; TP = tender points.

grupo-controle continuaram apenas o tratamento medicamentoso, mantendo também a rotina diária, inclusive de trabalho. Na avaliação ao término do tratamento e após 16 semanas ocorreu melhora estatisticamente significante no grupo experimental nos escores de FIQ, Health Assessment Questionnaire (HAQ) e Arthritis Impact Measurement
Scales (AIMS1), na contagem de tender points e na Escala Visual Analógica (EVA) para os sintomas da FM (dor de cabeça, fadiga, distúrbio no sono e sintomas gastrintestinais). Os autores concluíram que a balneoterapia com lama quente e imersão em água termal trouxeram benefícios aos pacientes com FM, mesmo sendo mantida a rotina diária, 
e ressaltaram a boa aceitação da terapia, visto que nenhum paciente foi retirado do estudo, em relação à baixa tolerância a outras modalidades de tratamento com medidas físicas freqüentemente recomendadas para esses pacientes ${ }^{(37)}$.

Vários possíveis mecanismos são apontados como responsáveis pelos efeitos benéficos da talassoterapia e da balneoterapia nas doenças reumáticas e especificamente na $\mathrm{FM}^{(41)}$. A balneoterapia com águas termais parece ter seu efeito benéfico atuando na formação de radicais livres, modificando a atividade de algumas enzimas, como a superóxido-dismutase, que se presume estarem alteradas na $\mathrm{FM}^{(42,43)}$. Nesse sentido, Bagis et al. ${ }^{(42)}$ estudaram 85 mulheres com FM e 80 mulheres saudáveis e encontraram níveis séricos significantemente menores de superóxidodismutase naquelas com FM quando comparados com as mulheres do grupo-controle, levantando a hipótese da FM como uma desordem oxidativa.

Acredita-se que os banhos térmicos e os banhos de lama parecem melhorar os sintomas da FM em razão dos efeitos da pressão hidrostática e da temperatura, esta última atuando sobre o limiar de dor e o tônus muscular ${ }^{(44)}$. Outro mecanismo que tenta explicar a melhora clínica de pacientes com FM quando submetidos à balneoterapia é sua possível ação sobre os mediadores inflamatórios. Ardiç et al. ${ }^{(40)}$ estudaram a influência da balneoterapia realizada durante três semanas sobre os níveis séricos de marcadores inflamatórios em três grupos de pacientes randomizados, como segue: grupo l (12 mulheres com FM realizaram balneoterapia), grupo 2 (12 mulheres com FM não realizaram o tratamento) e grupo 3 (10 mulheres saudáveis não realizaram o tratamento). Todos os parâmetros bioquímicos e clínicos foram avaliados antes e após a intervenção. Os autores observaram, além da melhora nos escores dos parâmetros clínicos (EVA, FIQ, BDI) no grupo 1 em relação ao grupo 2 , que o nível sérico de PGE2 dos pacientes com FM era maior quando comparado ao grupo de mulheres saudáveis antes do tratamento e diminuiu durante a intervenção apenas no grupo 1. Observaram ainda que os níveis séricos de IL-1 e $\mathrm{LTB}_{4}$ também diminuíram após a balneoterapia no grupo tratado, sugerindo a influência de mediadores inflamatórios nos sintomas da FM.

A exemplo do que ocorre com o tratamento de outras doenças em reumatologia, os possíveis benefícios da talassoterapia e/ou da balneoterapia em FM ainda carecem de comprovação. Apesar de a maioria dos estudos selecionados para esta revisão serem controlados e randomizados ${ }^{(35-40)}$, falhas metodológicas são observadas em alguns estudos. O pequeno tamanho da amostra é verificado em estudos que chegam a ter apenas 10 pacientes compondo cada grupo $^{(34,39,40)}$. Alguns estudos apresentam aparentemente um tamanho amostral adequado ${ }^{(16,36,38)}$, porém a falta de descrição do cálculo da amostra impossibilita saber sua representatividade. Outra falha observada nesses estudos é o não detalhamento dos procedimentos realizados, o que dificulta sua reprodutibilidade.

No entanto, é a heterogeneidade nos desenhos dos estudos, o maior empecilho para uma análise conclusiva sobre a eficácia desses métodos, como segue: utilização de diferentes instrumentos na avaliação, diferença no tempo de duração do tratamento (10 a 30 dias) e de intervenção diária (25 minutos a seis horas). Ainda, os protocolos associam várias intervenções simultâneas, como talassoterapia, balneoterapia, exercícios físicos e orientação educacional e diversas modalidades da mesma intervenção (balneoterapia com banhos em piscina aquecida, água do mar, enxofre, aplicação de argila e algas) ${ }^{(34-37)}$. Cabe destacar que, na maioria dos estudos, os pacientes do grupo experimental ficam hospedados em ambientes confortáveis como resorts ou SPAs luxuosos, localizados em praias paradisíacas, com folga de algumas semanas da vida cotidiana, sendo comparados a indivíduos que permanecem na rotina diária.

Embora não se tenha conclusão definitiva sobre a real eficácia da talassoterapia e da balneoterapia, essa abordagem terapêutica tem sido utilizada freqüentemente e com benefícios na FM em vários países. Apesar de o nosso imenso litoral banhado pelo Atlântico e temperatura das águas adequada para a talassoterapia na maior parte do ano, esta não é utilizada no Brasil. Apenas grupos isolados realizaram estudos não-controlados, com poucos pacientes, usando banhos de imersão e exercícios na água do mar com resultados aparentemente satisfatórios ${ }^{(45,46)}$. As condições geográficas favoráveis do litoral brasileiro, aliadas ao possível benefício desta modalidade terapêutica na FM, justificam a realização de estudos com protocolos bem conduzidos (cálculo estatístico do tamanho amostral, controlado, randômico, instrumentos de coleta de dados validados, descrição minuciosa dos procedimentos e análise estatística adequada), no intuito de avaliar o potencial da talassoterapia como alternativa de tratamento em nosso meio. 


\section{REFERÊNCIAS}

1. Wolfe F, Smythe HA, Yunus MB et al.: The American College of Rheumatology 1990 criteria for classification of fibromyalgia: report of the multicenter criteria committee. Arthritis Rheum 33: 160-72, 1990.

2. Bennett RM, Clark SR, Goldberg L et al.: Aerobic fitness in patients with fibrositis. A controlled study of respiratory gas exchange and 133xenon clearance from exercising muscle. Arthritis Rheum 32: 454-60, 1989.

3. Valim V, Oliveira LM, Suda AL et al.: Peak oxygen uptake and ventilatory anaerobic threshold in fibromyalgia. J Rheumatol 29: 353-7, 2002.

4. Kaplan RM, Schmidt SM, Cronan TA: Quality of well being in patients with fibromyalgia. J Rheumatol 27:785-9, 2000.

5. Wolfe F, Anderson J, Harkness D et al.: Health status and disease severity in fibromyalgia. Arthritis Rheum 40:1571-9, 1997.

6. Gowans SE, deHueck A, Voss S, Richardson MA: Randomized, controlled trial of exercise and education for individuals with fibromyalgia. Arthritis Care Res 12:120-8, 1999.

7. Burckhardt CS, Mannerkorpi K, Hedenberg L, et al.: A randomized, controlled Clinical trial of education and physical training for women with fibromyalgia. J Rheumatol 21:714-20, 1994.

8. Valim V: Benefícios dos Exercícios Físicos na Fibromialgia. Rev Bras Reumatol 46:9-55, 2006.

9. Richards SC, Scott DL: Prescribed exercise in people with fibromyalgia: parallel group randomized controlled trial. BMJ 27:185-6, 2002 .

10. Mannerkorpi K: Exercise in fibromyalgia. Curr Opin Rheumatol 17:190-4, 2005.

11. Silva GD, Lage LV: Ioga na fibromialgia. Rev Bras Reumatol 46:37-9, 2006.

12. Gimenes RO, Santos EC, Silva TJP: Watsu no tratamento da fibromialgia: estudo piloto. Rev Bras Reumatol 46:75-6, 2006.

13. Fitzcharles M, Esdaile J: Nonphysician practitioner treatment and fibromyalgia syndrome. J Rheumatol 24:937-40, 1997.

14. Morris CR, Bowen L, Morris AJ: Integrative Therapy for Fibromyalgia: Possible Strategies for an Individualized Treatment Program. South Med J 98:177-84, 2005.

15. Taggart HM, Arslanian CL, Bae S, et al.: Effects of T'ai Chi Exercise on Fibromyalgia Symptoms and Health-Related Quality of Life. Orthop Nurs 22:353-60, 2003.

16. Buskila D, Abu-Shakra M, Neumann L, et al.: Balneotherapy for fibromyalgia at the Dead Sea. Rheumatol Int 20:105-8, 2001.

17. Wigler I, Elkayam O, Paran D, et al.: Spa therapy for gonarthrosis: a prospective study. Rheumatol Int 15:65-8, 1995.

18. Kovacs I, Bender T: The therapeutic effects of Cserkeszölö thermal water in osteoarthritis of knee: a double blind, controlled, follow up study. Rheumatol Int. 21:218-21, 2002.

19. Bender T, Karagülle Z, Bálint GP, et al.: Hydrotherapy, balneotherapy, and spa treatment in pain management. Rheumatol Int 25:220-4, 2004.

20. van Tubergen A, Landewé R, van der Heijde D, et al.: Combined spa-exercise therapy is effective in patients with ankylosing spondylitis: a randomized controlled trial. Arthritis Rheum $45: 430-8,2001$
21. Sukenik S, Buskila D, Neuman L, et. al: Sulphur bath and mud pack treatment for rheumatoid arthritis in the Dead Sea area. Ann Rheum Dis 49:99-102, 1990.

22. Moses SW, David M, Goldhammer E, et al.: The Dead Sea, A Unique Natural Health Resort. Isr Med Assoc J 8: 483-8, 2006.

23. Ortiz MR: Origenges y fundamentos de la talassoterapia. Revista de la Facultad de Ciencias de la Salud. 2:1-12, 2004.

24. Wolenski L, Fortuna A: Rheumatoid arthritis and the marine climate Minerva Med. 22:2497-500, 1980.

25. Franke A, Reiner L, Resch KL: Long-term benefit of radon spa therapy in the rehabilitation of rheumatoid arthritis: a randomised, double-blinded trial. Rheumatol Int 27: 703-13, 2007.

26. Jajic I: Balneotherapy and heliomarinotherapy in the treatment and rehabilitation of patients with psoriatic arthritis. Reumatizam 31:13-6, 1984.

27. Giardino G: Thermal thalassotherapy for arthropathic psoriasis and psoriasis in the aged. G Gerontol 14:441-6, 1966.

28. Altan L, Bingol U, Aslan M, et al.: The effect of balneotherapy on patients with ankylosing spondylitis. Scand J Rheumatol 35: 283-9, 2006.

29. Codish S, Dobrovinsky S, Abu Shakra M, et al.: Spa therapy for ankylosing spondylltis at the Dead Sea. Isr Med Assoc J 7: 443-6, 2005.

30. Evcik D, Kavuncu V, Yeter A, et al.: The efficacy of balneotherapy and mud-pack therapy in patients with knee osteoarthritis. Joint Bone Spine 74:60-5, 2007.

31. Verhagen A, Bierma-Zeinstra S, Boers M, et al.: Balneotherapy for osteoarthritis. Cochrane Database Syst Rev 17, 2007.

32. Constant F, Guillemin F, Collin JF, et al.: Use of spa therapy to improve the quality of life of chronic low back pain patients. Med Care 36:1309-14, 1998.

33. Pittler $M H$, Karagülle $M Z$, Karagülle $M$, et al.: Spa therapy and balneotherapy for treating low back pain: meta-analysis of randomized trials. Rheumatology 45:880-4, 2006.

34. Sukenik S, Baradin R, Codish C, et al.: Balneotherapy at the Dead Sea area for patients with psoriatic arthritis and concomitant fibromyalgia. Isr Med Assoc J 3:147-50, 2001.

35. Evcik D, Kizilay B, Gökçen E: The effects of balneotherapy on fibromyalgia patients. Rheumatol Int 22:56-9, 2002.

36. Zijlstra TR, van de Laar MA, Bernelot Moens HJ, et al.: SPA treatment for primary fibromyalgia syndrome: a combination of thalassotherapy, exercise and patient education improves symptoms and quality of life. Rheumatology 44: 539-46, 2005.

37. Fioravanti A, Perpignano G, Tirri G, et al.: Effects of mud-bath treatment on fibromyalgia patients: a randomized clinical trial. Rheumatol Int 27:1157-61, 2007.

38. Neumann L, Sukenik S, Bolotin A, et al.: The effect of balneotherapy at the Dead Sea on the quality of life of patients with fibromyalgia syndrome. Clin Rheumatol 20:15-9, 2001.

39. Donmez A, Karagulle MZ, Tercan N, et al.: SPA therapy in fibromyalgia: a randomised controlled clinic study. Rheumatol Int 26:168-72, 2005.

40. Ardiç F, Ozgen M, Aybek H, et al.: Effects of balneotherapy on serum IL-1, PGE2 and LTB4 levels in fibromyalgia patients. Rheumatol Int 27:441-6, 2007. 
41. Sukenik S, Flusser D, Abu-Shakra M: The role of spa therapy in various rheumatic diseases. Rheum Dis Clin North Am 25:883-97, 1999.

42. Bagis S, Tamer L, Sahin G, et al.: Free radicals and antioxidants in primary fibromyalgia: an oxidative stress disorder? Rheumatol Int 25:188-90, 2005.

43. Bender T, Bariska J, Vághy R, et al.: Effect of balneotherapy on the antioxidant system: a controlled pilot study. Arch Med Res 38:86-9, 2007.
44. Schmidt KL: Scientific basis of spa treatment in rheumatic diseases. Rheumatol Europe 24:136-40, 1995.

45. Almeida AB, Oliveira AMB; Bezerra ETA et al.: Talassoterapia. Anais do $2^{\circ}$ Congresso Brasileiro de Extensão Universitária, Belo Horizonte, 12 de setembro de 2004.

46. Balga RSM, Rocha CRO, Fonseca TS: Talassoterapia: melhoria da qualidade de vida na praia. Rev Mackenzie Ed Fisica Esporte 5: 119-126, 2006. 